\title{
Cerebellar Hemorrhage Secondary to Cranial Metastasis of Prostate Cancer
}

\author{
-Case Report- \\ Joji InAmasu, Yoshiki NAKAMURA, Ryoichi SAITO, Yoshiaki KUROSHIMA, \\ Keita MAYANAGI, Maaya ORII, and Kiyoshi ICHIKIZAKI
}

Department of Neurosurgery, National Tokyo Medical Center, Tokyo

\begin{abstract}
A 77-year-old man with a 9-year history of prostate cancer presented with high fever and dysphagia. The initial diagnosis was aspiration pneumonia, but the patient became comatose 2 days after admission, and neuroradiological workup revealed cerebellar hemorrhage, obstructive hydrocephalus, and extensive destruction of the occipital bone secondary to cranial metastasis. The diagnosis was cerebellar hemorrhage secondary to cranial metastasis of prostate cancer. Tumor resection was abandoned because of the patient's poor health. Shunt surgery and palliative radiotherapy were temporarily effective in restoring his consciousness, but he died of systemic infection 3 weeks after surgery. Metastasis of prostate cancer to the cranium, particularly to the skull base, rarely causes lower cranial nerve paresis, and awareness of this sign may lead to earlier detection of the cranial metastasis and prevention of cerebellar hemorrhage.
\end{abstract}

Key words: cerebellar hemorrhage, cranial metastasis, lower cranial nerve paresis, prostate cancer

\section{Introduction}

Prostate cancer is one of the solid neoplasms that tend to metastasize to bones throughout the entire body, including the skull. ${ }^{10,12)}$ The pelvis and vertebral column are most frequently affected, and cranial metastasis is relatively common with an incidence of $7 \%$ in patients with a known diagnosis of prostate cancer. ${ }^{10)}$ The occipital bone is the most frequently affected bone in the cranium, probably because of tumor cell seeding via Batson's vertebral venous plexus. ${ }^{11)}$ Cranial metastasis can usually be identified in symptomatic patients who complain of bone pain or a palpable mass, or with signs of cranial nerve paresis, and in asymptomatic patients with positive scintigraphic evidence. ${ }^{7,10,12)}$ Cranial metastases of prostate cancer are usually slowgrowing, osteoblastic masses, ${ }^{7)}$ and rarely cause acute deterioration. We report a case of cerebellar hemorrhage secondary to cranial metastasis of prostate cancer in an elderly patient with a long history of the disease who presented with dysphagia and pneumonia.

Received December 3, 2002; Accepted September 16, 2003

\section{Case Report}

A 77-year-old man with a 9-year history of prostate cancer came to our emergency room complaining of repeated vomiting and high fever. His prostate cancer (stage C, i.e., cancers involving soft tissues outside the prostate, but without remote metastasis ${ }^{9}$ ) had been successfully treated by hormone therapy at another hospital, and there had been no clinical signs of local recurrence or remote metastasis. He had experienced difficulty in swallowing and occasional occipital pain for several months, but had not sought medical attention. He suffered repeated vomiting 1 day before admission. Initially, a primary care physician made a diagnosis of aspiration pneumonia.

On admission the patient was drowsy, but was able to talk and obey commands. Hoarseness and dysphagia suggested the presence of lower cranial nerve paresis. He had no history of hypertension, but his blood pressure was 162/94 mmHg. The patient was admitted for treatment of pneumonia, but 2 days later, he became comatose.

A neurosurgical consultation was requested after computed tomography (CT) of the brain revealed cerebellar hemorrhage and extensive destruction 

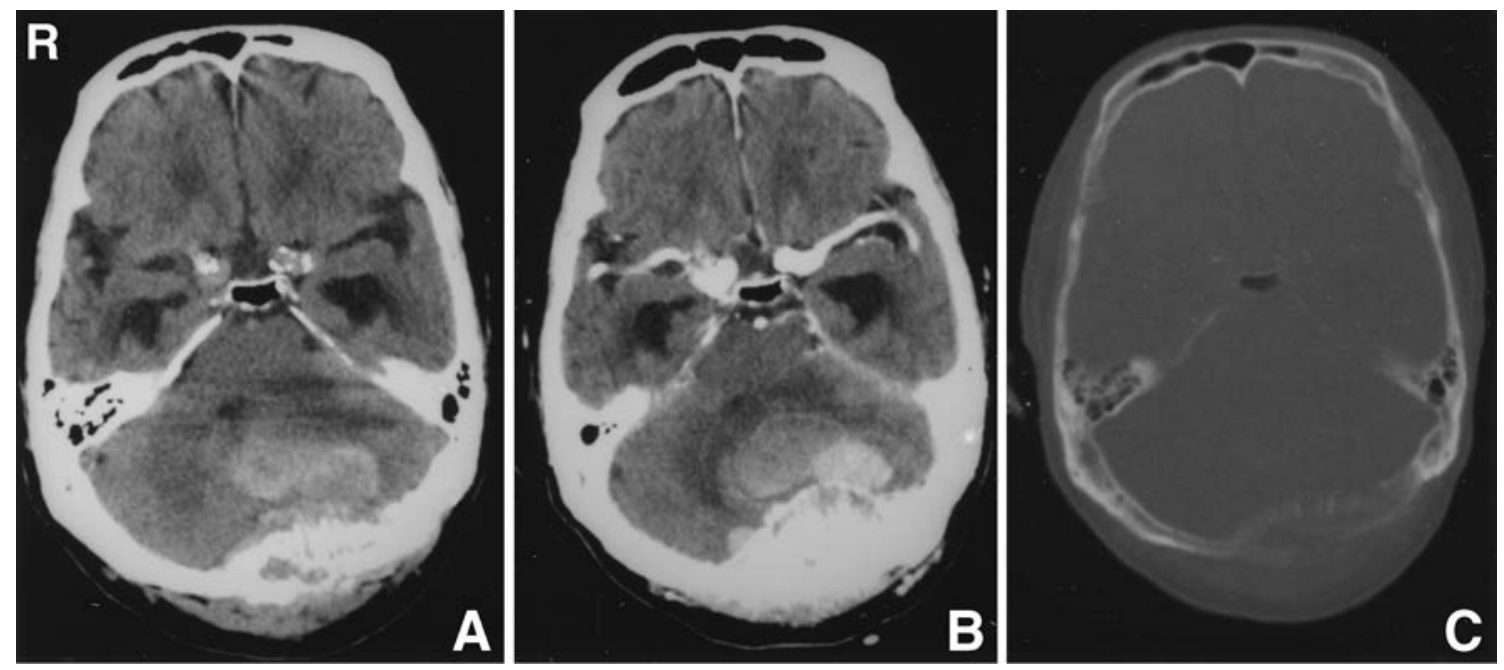

Fig. 1 Brain computed tomography scans showing cerebellar hemorrhage (A), extensive destruction and hyperostosis of the occipital bone (C: bone-window scan), and an enhanced mass adjacent to the hemorrhage, indicating the presence of an intradural tumor (B: scan with contrast medium).
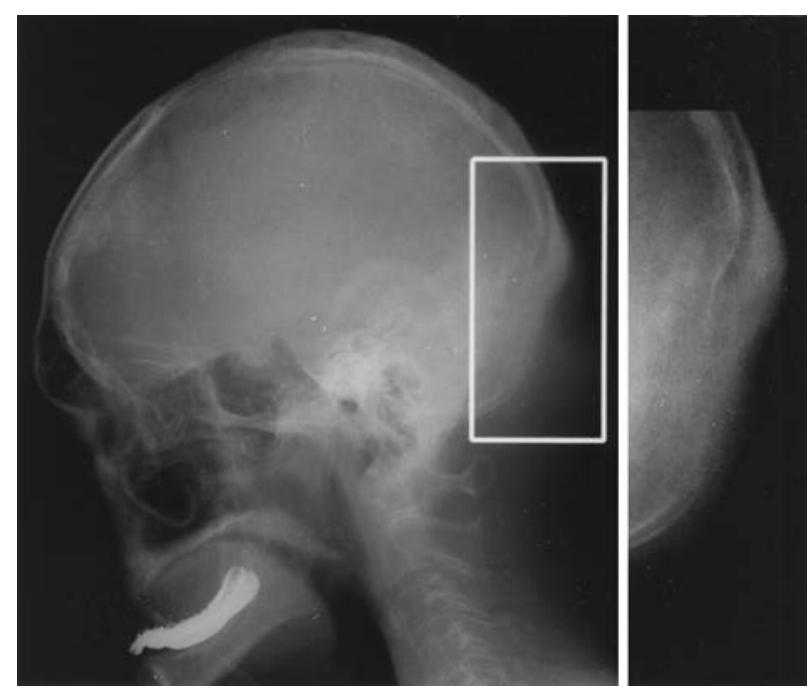

Fig. 2 Lateral skull radiographs showing extensive destruction and hyperostosis of the occipital bone, which is clearly observed in the magnified view (right).

and hyperostosis of the occipital bone (Fig. 1A, C). Palpation detected a hard, immobile mass over the occiput. CT with contrast medium revealed an enhanced mass adjacent to the hemorrhage, indicating the presence of a tumor (Fig. 1B). The cerebral ventricles were markedly dilated, indicating the presence of obstructive hydrocephalus. Skull radiography showed destruction and hyperostosis of the occipital bone (Fig. 2). Magnetic resonance imaging showed both the tumor and subacute hemorrhage, and subcutaneous invasion of the tumor (Fig. 3). The serum prostate-specific antigen level was elevated to $964 \mathrm{ng} / \mathrm{ml}$ (normal $4-10 \mathrm{ng} / \mathrm{ml}$ ). Both the platelet count and the coagulation function were normal. The diagnosis was cranial metastasis of prostate cancer resulting in invasion of the posterior fossa and cerebellar hemorrhage. Bone scintigraphy with technetium-99m showed several other metastases in the ribs (Fig. 4).

Resection of the tumor and the hemorrhage was planned, but later abandoned because of the patient's poor respiratory status. A ventriculoperitoneal shunt was implanted, followed by palliative radiotherapy. He regained consciousness after surgery, but developed a systemic infection and died 3 weeks postoperatively. Autopsy was not performed.

\section{Discussion}

The presence of hyperostosis with or without osteolysis is a characteristic finding of skull metastases of prostate cancer on radiography and CT. ${ }^{7)}$ Intracranial extension of cranial metastases as a result of penetration of the dura mater is rare, but cases mimicking meningiomas radiologically have been reported.6) The present case is unusual in the occurrence of the cerebellar hemorrhage. Intracerebral hemorrhage (ICH) secondary to metastasis of prostate cancer is rare, and the ICH was caused by intracranial metastasis in all reported cases. ${ }^{1-3)}$ The 

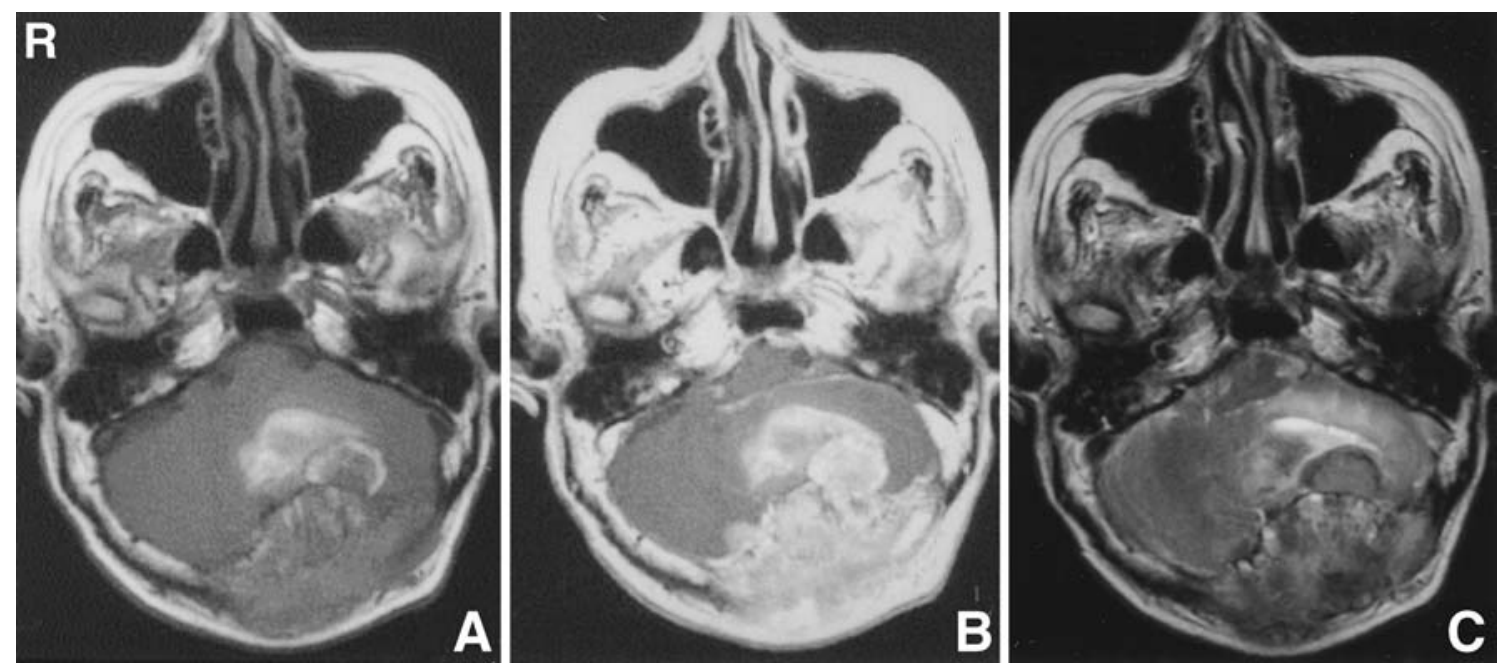

Fig. 3 Magnetic resonance images showing the tumor and the hemorrhage in the subacute stage appearing as high intensity on the $T_{1}$-weighted (A) and $T_{2}$-weighted (C) images, strong enhancement of the tumor by contrast medium (B), and extensive subcutaneous invasion of the tumor.

rarity may be due to both the very low incidence of brain metastasis of prostate cancer, with reported incidences of $0.4 \%$ and $0.6 \%$ in all patients with prostate cancer, ${ }^{4,8)}$ and to the relatively slow-growing, biological characteristics of prostate cancer. ${ }^{7,10)}$ Cranial metastasis is more frequently encountered than intracranial metastasis, but most patients present with complaints of bone pain and signs of cranial nerve paresis. ${ }^{7,10,12)}$ No case of cranial metastasis of prostate cancer associated with ICH could be found in a MEDLINE search.

The cause of the hemorrhage in the present case remains unknown, because no biopsy or autopsy was performed. The patient had no history of hypertension and hypertensive hemorrhage was considered unlikely. The coagulation function was within normal limits, and disseminated intravascular coagulopathy was also unlikely. Intratumoral hemorrhage may have been the cause, but the location of the hemorrhage was unusual in that it was adjacent to the solid tumor rather than in the center. Dural sinus occlusion by the tumor and subsequent compromise of venous outflow resulting in venous hypertension and hemorrhagic infarction is another possibility, although not confirmed angiographically.

The prognosis for patients with prostate cancer and cranial metastasis is grim compared to that for patients with a solitary vertebral metastasis. ${ }^{7,10,12)}$ The poor pulmonary performance of our patient precluded radical removal of the tumor. Prostate cancer is frequently radiosensitive, and palliative radiation therapy has been frequently recommended for prostate cancer patients with cranial metastasis. $^{5-7,11)}$ Shunt surgery followed by palliative radiotherapy was temporarily effective in restoring the patient's level of consciousness, but did not affect the outcome or prolong survival.

Metastasis of prostate cancer to the cranium, particularly to the skull base, may cause lower cranial nerve paresis and awareness of this sign may lead to earlier detection of the cranial metastasis and prevention of the secondary hemorrhage.

\section{References}

1) Barolat-Romana G, Maiman D, Dernbach P, Choi H: Prostate carcinoma presenting as intracranial hemorrhage. Case report. J Neurosurg 60: 414-416, 1984

2) Bucci MN, Farhat SM: Metastatic adenocarcinoma of the prostate as a cause of subdural hematoma. J Urol 135: 803-804, 1986

3) Chang DS, Hwang SL, Howng SL, Chai CY: Prostatic carcinoma with brain metastasis presenting as a tumor hemorrhage. Kaohsiung J Med Sci 14: 247-250, 1998

4) Chung TS, Thannikkary C: Carcinoma of the prostate with brain metastasis. J Surg Oncol 33: 103-105, 1986

5) Green JSA, Tomkinson A, Matanhelia SS, Peeling WB: Prostatic cancer presenting as dysphagia. Br J Clin Pract 48: 164-165, 1994

6) Kwee IL, Nakada T, St John JN: Triple fossa metastasis of prostate cancer. Neurosurgery 13: 584-586, 1983

7) Long MA, Husband JES: Features of unusual metastases from prostate cancer. Br J Radiol 72: 933-941, 1999 


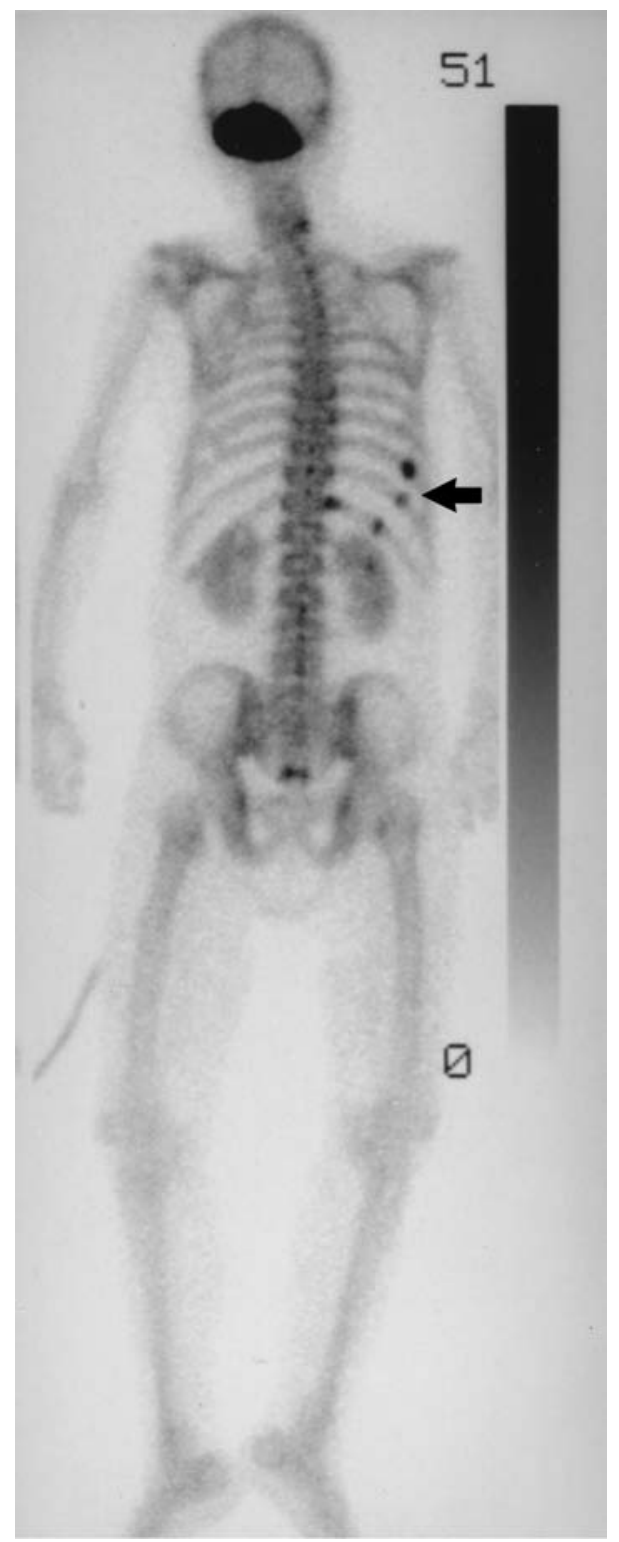

Fig. 4 Bone scintigram with technetium-99m showing an intense "hot spot" in the occipital bone, and several small metastases in the ribs (arrow).
8) McCutcheon IE, Eng DY, Logothetis CJ: Brain metastasis from prostate carcinoma: antemortem recognition and outcome after treatment. Cancer 86: 2301-2311, 1999

9) Narayan P: Neoplasm of the prostate gland, in Tanagho EA, McAninch JW (eds): Smith's General Urology, ed 14. Norwalk, Appleton \& Lange, 1995, pp 392-433

10) Rana A, Chisholm GD, Khan M, Sekharjit SS, Merrick MV, Elton RA: Patterns of bone metastasis and their prognostic significance in patients with carcinoma of the prostate. Br J Urol 72: 933-936, 1993

11) Sahin AA, Ro JY, Ordonez NG, Luna MA, Weber RS, Ayala AG: Temporal bone involvement by prostatic adenocarcinoma: report of two cases and review of the literature. Head Neck 13: 349-354, 1991

12) Saito $H$, Hida $M$, Shimbo $T$, Nakamura K, Yamagata J, Sato T: Metastatic patterns of prostatic cancer: correlation between sites and number of organ involved. Cancer 54: 3078-3084, 1984

Address reprint requests to: J. Inamasu, M.D., Department of Neurosurgery, National Tokyo Medical Center, 2-5-1 Higashigaoka, Meguro-ku, Tokyo 152-8902, Japan. e-mail: GInamasu@aol.com 